\section{Military Technical College Kobry El-Kobbah, Cairo, Egypt}

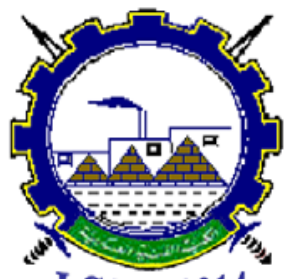

I.C.E.E.2014 $7^{\text {th }}$ International Conference
on

Chemical \& Environmental Engineering

27 - 29 May, 2014.

\title{
AGNM-2 \\ Nanotechnology Sensors, Systems, and Applications for Detecting Explosive
}

\begin{abstract}
One of the most significant current discussions in science of explosives is Detection of explosives. The number of terrorist attacks, and suicide bombings in particular, has risen dramatically in the last few years. Mitigation the worldwide explosive threat, both today and in the future is the strategic goal for detecting explosives materials (substances and mixtures). Early detection of hidden explosive devices therefore has the potential to save many lives. In order to achieve that goal it has to know the conventional and unconventional components of the explosive materials as well as the accurate characterization of their physical and chemical properties.
\end{abstract}

Tamer Z. Wafy ${ }^{*}$

As nanomaterials become more readily available for commercial devices, great interest has been shown in using them to develop trace-level detection systems for explosives which overcome all of these issues. Because of the unique nature and tuneable properties of nanomaterials like carbon nanotubes, nanowires, and other nanostructures, handheld or portable systems which are sensitive down to the molecular level could well be possible

The purpose of this lecture is to review recent research into the common threads form explosives materials, applications, requirements and challenges of detection technologies, existing systems which are capable of detecting compounds, nanotechnology of chemical sensors and sensor devices structure based on nanomaterials, nanocarbons (single wall nanotubes, multiwall nanotubes and graphene nanosheets), titania $\left(\mathrm{TiO}_{2}\right)$ nanotube array and nanoparticle systems (gold nanoparticles and silica nanoparticles). Finally the suggested recommendations for implementation of nanotechnology of chemical sensors are presented.

Control stage (Key performance indicators)

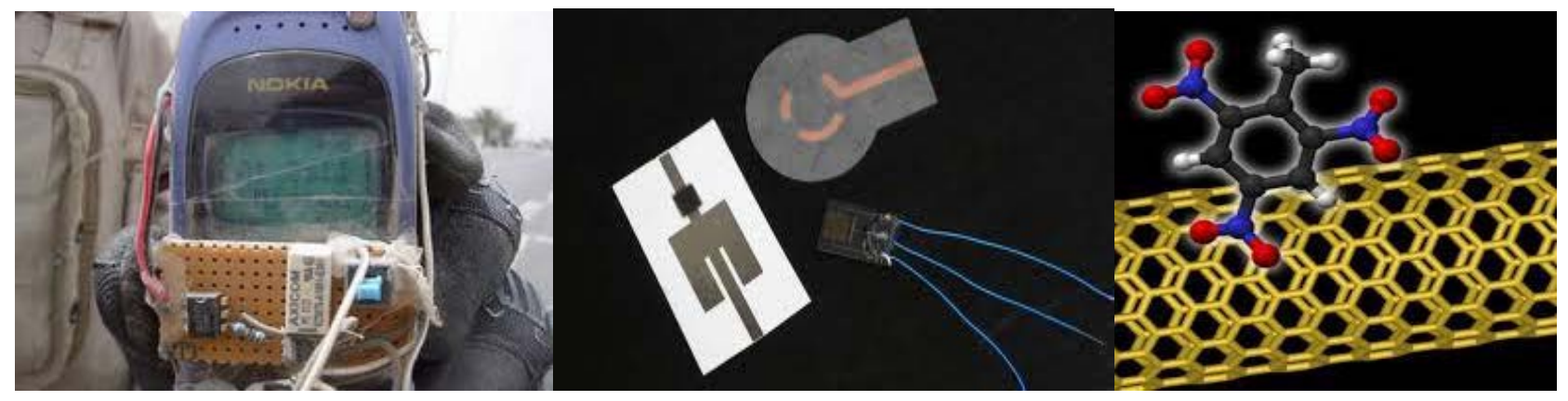

Egyptian Armed Forces 